"Vorlesungen über elektrische Ströme", as given in 1864-65, published with his permission in 1884 by K. Vondermühl, we find on p. 197 a careful reference to Gaugain (the only reference in a 36-page chapter) and no mention of Helmholtz.

This letter is written in the hope that some reader of NATURE may have additional evidence to offer. If none is forthcoming, I think we should call the two-coil combination Gaugain-Helmholtz coils. If the whole truth were known, it seems probable that the proper designation would be Gaugain-Neumann. L. W. McKmehaN.

Sloane Physical Laboratory,

Yale University, New Haven, Conn. April 11.

\section{The Apparent Thermionic Constant $A$ of Clean Metals}

THERE seems to be little room for doubt that the apparent thermionic $A$ (the $A$ derived from a Richardson line) of at least some clean metals is genuinely less than the upper theoretical limit for this quantity, $A_{0}$, the value of which is $120 \mathrm{amp}$. em..$^{-2}$ deg..$^{-2}$. Thus, for tungsten, tantalum and molybdenum, which are among the metals for which the most reliable data are available, values of $60-100$, 60 and $55 \mathrm{amp}$. cm. $.^{-2} \mathrm{deg} .^{-2}$ respectively have been obtained, and, if surface roughness had been taken into account, somewhat smaller values still must have been found. It would, of course, be quite possible to attribute these results to an imperfect transmission of the electrons through the emitting surfaces, assuming the apparent $A$ to be the true $A$. There is, however, a known effect which, unless compensated for by other effects, must make the apparent $A$ less than the true $A$ by a factor of at least 2 or 3 , and which would, therefore, account for the order of magnitude of the observed data on the assumption of practically perfect transmission.

It is well known that the apparent $A$ will differ from the true $A$ if the work function $\chi$ varies with the temperature. According to Sommerfeld's theory of metals, this quantity is given by the equation

$$
\chi=C-\frac{h^{2}}{8 m}\left(\frac{3 n}{\pi}\right)^{2 / 3}
$$

where $C, h, m$ and $n$ are the product of the inner potential and the electronic charge, Planck's quantum of action, the electronic mass, and the number of effectively free electrons per unit volume of the metal respectively. Strictly speaking, there should be a further term in the expression for $\chi$, but with ordinary free electron concentrations this is small and its temperature dependence makes the apparent $A$ differ from the true $A$ by only something like 2 per cent. Apart from this, $\chi$ might vary with the temperature, as Fowler ${ }^{1}$ has pointed out, owing to a temperature variation either of $C$ or of $n$. Concerning what variation of $C$ with temperature is to be expected, little, unfortunately, seems to be known. There must, however, be a temperature variation of $n$ associated with the thermal expansion of the metal, if, as is probable, the number of free electrons per atom remains constant. It appears worth while, then, to see whether the experimental data might be accounted for by assuming that $C$ is sufficiently nearly constant for the temperature variation of $\chi$ to be determined in direction and order of magnitude by that of $n$ alone.
Let the coefficient of linear expansion be denoted by $\alpha$. Then from the formula for $\chi$ we find that the corresponding factor, $f$, by which the apparent $A$ must be less than the true $A$ is given by

$$
f=\exp .\left\{\frac{h^{2} \alpha}{4 m k}\left(\frac{3 n}{\pi}\right)^{2 / 3}\right\} .
$$

In the temperature regions where thermionic measurements are usually made, $\alpha$ has the value $6 \cdot 3 \times$ $10^{-8}$ deg..$^{-1}$ for tungsten and $8.0 \times 10^{-6} \mathrm{deg} .^{-1}$ for both tantalum and molybdenum. On the assumption of one free electron per atom the values of $n$ for the three metals in the order named would be $6 \cdot 2 \times 10^{22}$, $5.6 \times 10^{22}$ and $6.3 \times 10^{22}$ per $\mathrm{cm}^{3}$ respectively. Hence for the corresponding values of $f$ we should have $2 \cdot 3,2 \cdot 7$ and $2 \cdot 9$ respectively. For two free electrons per atom we should have, instead, $3 \cdot 7,4 \cdot 3$ and $4 \cdot 6$ respectively. These are of the order of magnitude of the factors by which the apparent $A$-values fall short of $A_{0}$.

Research Laboratories of the

\section{A. L. Reimann.}

General Electric Company, Ltd., Wembley.

April 30.

${ }^{1}$ R. H. Fowler, Proc. Roy. Soc., A, 122, 36; 1929.

\section{Isomeric Nuclei?}

As I have shown elsewhere ${ }^{1}$, the introduction of negative protons into nuclear structure leads to the possibility of the existence of isomeric nuclei, that is, nuclei with the same atomic number and atomic weight but different internal structure (a pair of positive and negative protons instead of a pair of neutrons). As an example, the nucleus of uranium.Z was given, which seems to be isomeric with uranium$\mathrm{X}_{2}$.

A further indication is furnished by recent measurements of Aston ${ }^{2}$, who has found in the mass-spectra of ordinary lead the line 210. This isotope of lead has the same atomic number and atomic weight as radium-D but, since it exists to the extent of 0.08 per cent, it cannot be, of course, the usual radioactive radium-D. Therefore, if the ascription of the observed line to lead is not erroneous, we must conclude that the nucleus with atomic number 82 and atomic weight 210 may exist in two modifications (isomers) of which one is stable and the other is subject to $\beta$-decay.

$$
\begin{gathered}
\text { Institute for Theoretical Physics, } \\
\text { Copenhagen. } \\
\text { April 25. }
\end{gathered}
$$

1 G. Gamow, Phys. Rev. (in print).

${ }^{2}$ F. W. Aston, Proc. Roy. Soc., A, 140, $535 ; 1933$.

\section{Ground State of $\mathrm{C}_{2}$ and $\mathrm{O}_{2}$ and the Theory of Valency}

ACCORDING to the quantum mechanical theory of the chemical bond in its original form, the lowest state of a diatomic molecule should be a singlet term. The ground states of $\mathrm{C}_{2}\left({ }^{3} \Pi_{u}\right)$ and $\mathrm{O}_{2}\left({ }^{3} \Sigma_{g}^{-}\right)$found experimentally seem to be in contradiction with this theory while other considerations (Hund, Mulliken, Lennard-Jones) lead to the right result.

But it can be seen that the above mentioned theory also easily explains the experimental facts. One has only to take into account that the binding 